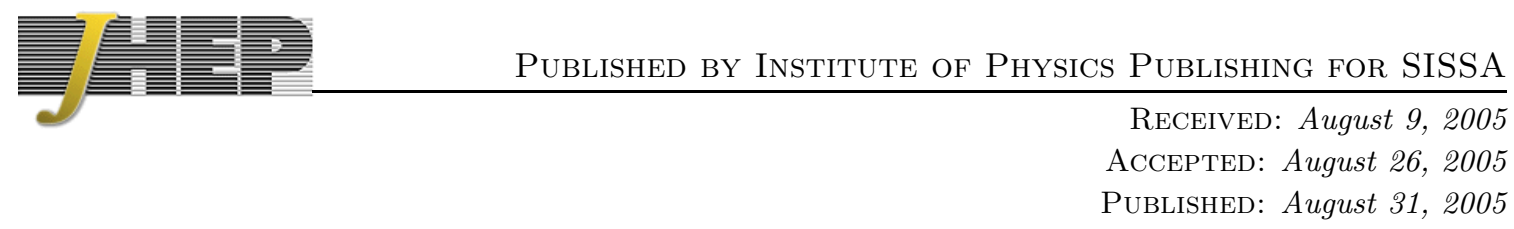

\title{
A note on D1-D5-J system and 5D small black ring
}

\author{
Norihiro lizuka $^{a}$ and Masaki Shigemori ${ }^{b}$ \\ ${ }^{a}$ Tata Institute of Fundamental Research \\ Homi Bhabha Road, Mumbai 400 005, India \\ ${ }^{b}$ California Institute of Technology 452-48 \\ Pasadena, CA 91125, U.S.A. \\ E-mail: iizuka@theory.tifr.res.in, shige@theory.caltech.edu
}

ABSTRACT: The "small" black ring in 5D obtained by giving angular momentum to the D1-D5 system compactified on $S^{1} \times \mathrm{K} 3$ is a very interesting object in the sense that it does not have an event horizon in the supergravity limit whereas it microscopically has a finite entropy. The microscopic origin of this small black ring can be analyzed in detail since it is constructed by adding angular momentum to the well-studied D1-D5 system. On the other hand, its macroscopic, geometrical picture is difficult to study directly. In this note, by duality transformations and the $4 \mathrm{D}-5 \mathrm{D}$ connection, we relate this $5 \mathrm{D}$ small black ring to a 4D small non-rotating black hole, where the latter is known to develop a non-vanishing horizon due to stringy $R^{2}$ corrections to the supergravity action. The entropy of the $4 \mathrm{D}$ small black hole agrees with the microscopic entropy of the 5D small black ring, which supports the validity of the 4D-5D connection even for small black objects. These results give an indirect evidence that a non-vanishing horizon is formed for the $5 \mathrm{D}$ small black ring.

Keywords: Black Holes in String Theory, D-branes. 


\section{Contents}

1. Introduction 1

2. D1-D5-J system 2

3. 4D-5D connection

4. Conclusion and discussion 7

\section{Introduction}

The D1-D5-P system, made of $N_{1}$ D1-branes, $N_{5}$ D5-branes and $N_{P}$ units of momentum, has been an ideal arena for studying microscopic physics of black holes in string theory [1]. The $N_{P}=0$ case (the D1-D5 system) is not classically a black hole because its horizon vanishes at the supergravity level, whereas its microscopic entropy computed from the dual CFT is finite: $S=2 \sqrt{2} \pi \sqrt{N}$ ( $T^{4}$ compactification) and $S=4 \pi \sqrt{N}$ (K3 compactification), where $N \equiv N_{1} N_{5}$. In a recent beautiful paper by Dabholkar [2], it was shown that heterotic $4 \mathrm{D}$ black hole with classically vanishing horizon, which is dual to the above D1-D5 system compactified on $T^{2} \times \mathrm{K} 3$, becomes a black hole with string-size event horizon once stringy $R^{2}$ corrections to the supergravity action are taken into account, and that the $R^{2}$-corrected macroscopic entropy ${ }^{1}$ of such "small" black hole agrees with the microscopic entropy, as predicted by Sen [4]. Further references for these developments include [ [5, 6].

If we consider the D1-D5 system compactified instead on $S^{1} \times \mathrm{K} 3$ and add angular momentum $J=\mathcal{O}(N)$ to it, the brane worldvolume starts to look like a ring [7, 8] from the 5D viewpoint, rather than a pointlike object. Therefore, this D1-D5-J system is expected to be described by a "small" version of the black ring in 5D supergravity [9], once we consider stringy corrections to the supergravity action. However, a systematic framework for studying stringy corrections to the 5D supergravity, like the one for 4D [3], has not been available so far, so we cannot directly study the stringy corrections to this system.

Recently, a new connection between 4D and 5D black objects was proposed [10-13], which relates the partition function of a 5D black hole/ring with that of a 4D black hole. Although this "4D-5D connection" is based on supergravity analysis, it is expected to hold even if we take into account stringy corrections, since it is natural to expect that a BPS solution interpolating 4D and 5D objects exists even if stringy corrections are included, and the continuous-moduli-independence of entropy is the property of the microscopic theory,

\footnotetext{
${ }^{1}$ The $R^{2}$-corrected macroscopic entropy was derived in a sequence of papers $[3]$.
} 
not of supergravity. Indeed, the microscopic entropy of 5D black ring [14, 15] agrees with that of 4D black hole [16] including quantum corrections [17, 18]. This suggests that $5 \mathrm{D}$ black holes/rings which classically have no horizon will generally develop finite small horizon.

Therefore, one naturally wants to apply the 4D-5D connection to the D1-D5-J ring system on $S^{1} \times \mathrm{K} 3$ to study its properties using $4 \mathrm{D}$ techniques. However, the 4D-5D connection is not directly applicable, since one cannot get to the duality frame in which one can make use of the 4D-5D connection via simple $S$-duality and $T$-duality along $S^{1}$ directions in type-II. In this note, we map by $U$-duality the D1-D5-J system into a duality frame in which the 4D-5D connection is applicable, and relate it with a small non-rotating black hole in 4D. The microscopic entropy counting is well-understood for both these 4D and 5D configurations. On the macroscopic side, the horizon geometry of the 4D small non-rotating black hole is well-understood whereas the geometry of the original D1-D5-J system, which is expected to be a 5D small black ring, is not well-understood. By connecting this D1-D5-J system with the geometry of the 4D small black hole that has a small horizon, we give an indirect evidence of event horizon showing up in the 5D small black ring by stringy corrections to the supergravity action.

The organization of this paper is as follows. In section 2, we study the microscopic entropy of the D1-D5-J system, and clarify the limit in which it can be regarded as a ring with a well-defined profile. In section 3, we present a duality chain which relates the D1-D5-J system and a 4D non-rotating small black hole via the 4D-5D connection. The entropy of the $4 \mathrm{D}$ small black hole agrees with the microscopic entropy of the D1-D5-J system, which justifies applying the 4D-5D connection for the small black objects. Also, this $4 \mathrm{D}$ small black hole has non-vanishing horizon because of stringy corrections to the supergravity action. These results suggest that event horizon appears for the 5D small black ring by stringy corrections to the supergravity action. In section \&, We conclude with some comments on future directions.

The D1-D5-J small black ring system and its entropy was also studied recently by Kraus and Larsen 19] from a different point of view.

\section{D1-D5-J system}

In order to understand the charges and dipole charges that appear in the D1-D5-J system, it is easiest to start from heterotic string compactified in $\mathbb{R}^{1,4} \times S^{1} \times T^{4}$. Let us take $\mathbb{R}^{1,4}, S^{1}$, and $T^{4}$ directions to be 01234,5 , and 6789 directions, respectively. We will write the $S^{1}$ as $S_{5}^{1}$ henceforth. We wrap $N_{F}$ F1's along $S_{5}^{1}$ and put on it $N_{P}$ units of linear momentum along $S_{5}^{1}$. From Virasoro constraint and BPS condition, we should impose $N_{L}=1-N_{F} N_{P}$, where $N_{L}$ is the left-moving oscillation number. Since $N_{L}>0$, we should choose $N_{F} N_{P}<0$. Furthermore, we give $J=n_{p}$ units of angular momentum to the system in the $\psi$ direction, where $\psi$ is the angular direction on the 1-2 plane. Now the F1 worldvolume is a helix or a coil wound on a cylinder spanned by $\psi$ and $x^{5}$, moving upwards along the $x^{5}$ axis. From the 5D (012345) point 
of view, the system looks like a ring in the $\psi$ direction. The configuration is as follows:

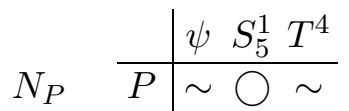

$$
\begin{aligned}
& N_{F} \quad F 1 \sim \bigcirc \sim \\
& n_{p} \quad p \bigcirc \sim \sim \\
& n_{f} \quad f 1 \bigcirc \sim \sim
\end{aligned}
$$

Here, " $\bigcirc$ " denotes wrapped directions, while " " denotes smeared directions. $P, p$ denote momenta and $N_{P}, n_{p}$ are the corresponding momentum numbers. $F 1, f 1$ denote fundamental string and $N_{F}, n_{f}$ are the corresponding winding numbers. The lowercase letters mean that they are along the contractible direction $\psi$. The momentum number $n_{p}$ along $\psi$ is nothing but angular momentum number $J$. We will refer to this system (2.1) as the FP system.

The maximum angular momentum we can have is $J_{\max }=N=\left|N_{F} N_{P}\right|=-N_{F} N_{P}$, which is the case when the F1 worldvolume is a perfect helix of radius $\sim \sqrt{J_{\max }}$. In the corresponding classical solution [7], the F1 winds once around the $\psi$ circle while it winds $N_{F}$ times the $x^{5}$ direction $S_{5}^{1}$. Therefore, $n_{f}=1$ if $J=J_{\max }$. For $J<J_{\max }$, the F1 worldvolume starts to fluctuate around the perfect helix, and it is not obvious that the winding number $n_{f}$ is well-defined. We will discuss later in what limit $n_{f}$ is well-defined.

The system (2.1) is dual to the well-studied D1-D5 system in type-IIB compactified on $S^{1} \times \mathrm{K} 3$, by the following chain of dualities: heterotic/IIA duality, $T(5)^{2}$, and then $S$. The resulting configuration is:

$$
\begin{array}{cc|ccc} 
& & \psi & S_{5}^{1} & \mathrm{~K} 3 \\
N_{1}=N_{P} & D 1 & \sim & \sim & \sim \\
N_{5}=-N_{F} & D 5 & \sim & \bigcirc \\
n_{p}=n_{p} & p & \bigcirc & \sim & \sim \\
n_{k k}=-n_{f} & k k & \bigcirc & S^{1} & \bigcirc
\end{array}
$$

Now the 6789 direction is K3, and " $S$ " means the special circle of the KK monopole. The numbers of D1- and D5-branes are $N_{1}=N_{P}$ and $N_{5}=-N_{F}$. Note that the relation between the F1 number in heterotic string and the NS5 number in type-IIA involves a minus sign [21, 22]. It is consistent with the fact that we need $N_{1} N_{5}>0$ for susy on the type-II side. This system is dual to a $1+1$ dimensional $\mathcal{N}=(4,4) \mathrm{CFT}$, which is sigma model with target space $\mathrm{K} 3^{N} / S_{N}, N=N_{1} N_{5}$ [23].

Now, let us count the entropy of the D1-D5-J system (2.2), or equivalently, the FP system (2.1). Let us here take the FP description, which is a special case of the computation done in [24]. We need to count the number of states generated by 24 left-moving transverse bosons $\alpha_{n}^{i}, i=1, \ldots, 24$, at level $N=\left|N_{F} N_{P}\right|=-N_{F} N_{P}$ that have angular momentum $J=n_{p}$ in the 1-2 plane.

\footnotetext{
${ }^{2} T$-duality along the axis of such helical objects was discussed in [20].
} 
If we write

$$
\alpha_{n}^{i=1}=\sqrt{\frac{n}{2}}\left(a_{n}^{+}+a_{n}^{-}\right), \quad \alpha_{n}^{i=2}=i \sqrt{\frac{n}{2}}\left(a_{n}^{+}-a_{n}^{-}\right), \quad n=1,2, \ldots,
$$

then the level $N$ and angular momentum $J$ are

$$
\begin{aligned}
& N=\sum_{n=1}^{\infty} \sum_{i=1}^{24} \alpha_{-n}^{i} \alpha_{n}^{i}=\sum_{n=1}^{\infty}\left[n\left(a_{n}^{+\dagger} a_{n}^{+}+a_{n}^{-\dagger} a_{n}^{-}\right)+\sum_{i=3}^{24} \alpha_{-n}^{i} \alpha_{n}^{i}\right] \\
& J=-i \sum_{n=1}^{\infty} \frac{1}{n}\left(\alpha_{-n}^{1} \alpha_{n}^{2}-\alpha_{-n}^{2} \alpha_{n}^{1}\right)=\sum_{n=1}^{\infty}\left[a_{n}^{+\dagger} a_{n}^{+}-a_{n}^{-\dagger} a_{n}^{-}\right]
\end{aligned}
$$

We can compute the entropy $S(N, J)$ by studying the partition function

$$
Z=\operatorname{Tr}\left[e^{-\beta(N+\lambda J)}\right]=\sum_{N, J} d_{N, J} q^{N} z^{J} .
$$

Here $\lambda$ is the chemical potential conjugate to $J$, and $q=e^{-\beta}, z=e^{\beta \lambda}$. The evaluation of $d_{N, J}$ was done in [24, and the leading term of the entropy for $N \gg 1, J=\mathcal{O}(N)$ is

$$
S=\log d_{N, J}=4 \pi \sqrt{N-|J|} .
$$

One sees from (2.7) that the only effect of $J \neq 0$ is to replace $N$ in the entropy formula with $N-J$. Here we assumed $J>0, J=\mathcal{O}(N)$. This means that, Bose-Einstein condensation of $J a_{n=1}^{+}$particles occurs and the whole angular momentum $J$ is carried by the condensate. The remaining particles have level $N-J$ but no net angular momentum. In other words, in the ensemble with level $N$ and angular momentum $J>0, J=\mathcal{O}(N)$, the states that contribute to entropy are of the form

$$
\underbrace{\left(a_{n=1}^{+}\right)^{J}}_{\begin{array}{l}
\text { Bose-Einstein } \\
\text { condensate }
\end{array}} \times \underbrace{\prod_{n=1}^{\infty}\left[\prod_{i= \pm, 3 \ldots 24}\left(\alpha_{-n}^{i}\right)^{N_{n i}}\right]|0\rangle}_{\begin{array}{l}
\text { states that are responsible for } \\
\text { entropy of the ensemble with } \\
\text { level } N-J \text { and no angular mo- } \\
\text { mentum }
\end{array}} .
$$

The state $\left(a_{n=1}^{+}\right)^{J}|0\rangle, J>0$ represents (classically) a fundamental string that goes once around the circle in the 1-2 plane of radius $\sim \sqrt{J}$, which is large if $J \gg 1$ [0]. Clearly, this state has $n_{f}=1$. Acting on this state by the operator $\prod_{n=1}^{\infty}\left[\prod_{i= \pm, 3 \ldots 24}\left(\alpha_{-n}^{i}\right)^{N_{n i}}\right]$ in (2.8) makes the fundamental string fluctuate around this circle. If this fluctuation is much smaller than the radius of the circle $\sim \sqrt{J}$, the winding number $n_{f}$ is well-defined and $n_{f}=1$. Because $\sum_{n i} N_{n i}=N-J$, statistical mechanics tells us that $N_{n i} \sim \sqrt{N-J}$ for $n=\mathcal{O}(1)$. This means that the size of the fluctuation is of order $(N-J)^{1 / 4}$, which is much smaller than the radius of the circle $\sqrt{J}$ if

$$
N, J \gg 1, \quad J=\mathcal{O}(N)
$$


In this limit, the system is expected to become a small black ring, ${ }^{3}$ and talking about winding number along $\psi$ makes sense, so we can say $n_{f}=1$. This relation between the black hole/ring transition and Bose-Einstein condensation is very reminiscent of the microscopic description of the large black ring in [14].

If $J<0$, then the above argument all goes through if we replace $\left(a_{n=1}^{+}\right)^{J}$ with $\left(a_{n=1}^{-}\right)^{|J|}$. In this case, $n_{f}=-1$. So, the $|J|$ in (2.7) can be replaced with $\left|n_{p}\right|=n_{f} n_{p}$.

Therefore, the entropy of the system (2.1) or (2.2) can be written as

$$
S_{\text {micro }}=4 \pi \sqrt{-N_{F} N_{P}-n_{f} n_{p}}=4 \pi \sqrt{N_{1} N_{5}+n_{p} n_{k k}},
$$

where in the last equality we used the relation between charges listed in (2.2).

The entropy counting can be done also in the D1-D5 frame (2.2) using the dual CFT. If we replace the oscillators $\alpha_{-n}^{i}$ with the chiral primaries of the $\mathcal{N}=(4,4) \mathrm{CFT}$ [26], the counting can be done in a completely identical way.

\section{3. $4 \mathrm{D}-5 \mathrm{D}$ connection}

Now, we would like use the 4D-5D connection 10-13 in order to deform the $5 \mathrm{D}$ small black ring (2.1) (or equivalently (2.2)) into a 4D small black hole.

The obstacle to doing that in a straightforward manner is that $T$-duality along the $S_{5}^{1}$ direction and $S$-duality in type-II string will not take the system (2.2) to a duality frame in which the 4D-5D connection as derived in [10-13] is applicable; one cannot avoid having unwanted nonzero charges. Fortunately, type-IIA string on $S^{1} \times \mathrm{K} 3$ has $O(5,21 ; \mathbb{Z})$ symmetry as a part of the $U$-duality group [27, 21] ${ }^{4}$, which interchanges charges so that we can use the 4D-5D connection. Or equivalently, we start from heterotic configuration (2.1), where we have $O(5,21 ; \mathbb{Z})$ symmetry as $T$-duality. After $T$-dualizing the charges appropriately in heterotic string, we can then go to type-II by heterotic/IIA duality.

Either way, after such a chain of dualities, we end up with the following configuration in type-IIA:

\begin{tabular}{rc|l} 
& & $\psi S_{5}^{1} \mathrm{~K} 3$ \\
\cline { 2 - 4 }$N_{P}$ & $D 2$ & $\sim \sim \alpha_{2}$ \\
$-N_{F}$ & $D 2$ & $\sim \sim \alpha_{3}$ \\
$n_{p}$ & $p$ & $\bigcirc \sim \sim$ \\
$-n_{f}$ & $n s 5$ & $\bigcirc \sim \bigcirc$
\end{tabular}

Here $\alpha_{a}$ are 2-cycles in K3, i.e., $\alpha_{a} \in H_{2}(\mathrm{~K} 3), a=2,3, \ldots, 23$ (the reason for reserving the index 1 will become clear shortly). The $F, P$ in (2.1) have been mapped into D2-branes wrapping particular two 2-cycles, $\alpha_{2}$ and $\alpha_{3}$.

Which particular two 2-cycles $\alpha_{2}, \alpha_{3}$ should the D2-branes wrap? This question can be answered as follows. First, let us recall how the $O(5,21 ; \mathbb{Z}) T$-duality group arises in

\footnotetext{
${ }^{3}$ More detailed analysis shows that, if $J=\mathcal{O}\left(N^{\gamma}\right), 1 / 2<\gamma \leq 1$, Bose-Einstein condensation occurs and the system is expected to become a small black ring in $5 \mathrm{D}$. On the other hand, if $\gamma \leq 1 / 2$, Bose-Einstein does not occur and the system is expected to become a small rotating black hole in 5D 25].

${ }^{4}$ The full U-duality group is $O(5,21 ; \mathbb{Z}) \times \mathbb{Z}_{2}$ 27, 21.
} 
heterotic string. In heterotic string theory on $S^{1} \times T^{4}$, there are 21 left- and 5 right-moving momenta in the internal directions. These momenta are quantized electric charges from the $5 \mathrm{D}$ point of view. They form the 26 -dimensional Narain lattice with signature $(5,21)$, and the $O(5,21 ; \mathbb{Z}) T$-duality rotates the charges in this lattice. $N_{P}$ and $N_{F}$ in (2.1) are two such electric charges of heterotic string, and in the 2-dimensional sublattice of the Narain lattice in which these charges live, the metric is proportional to $\left(\begin{array}{ll}0 & 1 \\ 1 & 0\end{array}\right)$ (recall that the left- and right-moving momenta in the $x^{5}$ direction are $p_{L, R}^{5}=N_{P} / R \pm N_{F} R / \alpha^{\prime}$, and the invariant form contains $\left(p_{L}^{5}\right)^{2}-\left(p_{R}^{5}\right)^{2}=4 N_{P} N_{F} / \alpha^{\prime}$ 28]). Correspondingly, there is $O(5,21 ; \mathbb{Z}) U$-duality group on the IIA side. This $O(5,21 ; \mathbb{Z})$ group contains $O(3,19 ; \mathbb{Z})$ subgroup that interchanges the D2-branes wrapping 2-cycles in K3. Because there are 22 2-cycles $\alpha_{a}, a=2, \ldots, 23$ in $\mathrm{K} 3$, the D2-brane charges live in 22-dimensional lattice $H_{2}(\mathrm{~K} 3, \mathbb{Z})$. The metric for this charge lattice is the intersection number $C_{a b}$ of 2-cycles in K3, which is known to have signature $(3,19)$ 29]. So, the answer to the question above is: we should choose the 2-cycles $\alpha_{2}, \alpha_{3}$ so that, in the 2-dimensional sublattice of the lattice $H_{2}(\mathrm{~K} 3, \mathbb{Z})$ in which they live, the metric should be $\left(\begin{array}{ll}0 & 1 \\ 1 & 0\end{array}\right)$. In other words, the intersection numbers $C_{a b}$ should satisfy

$$
C_{22}=C_{33}=0, \quad C_{23}=1 .
$$

Now we are ready to use the $4 \mathrm{D}-5 \mathrm{D}$ connection. Uplifting (3.1) to M-theory,

$$
\begin{array}{cc|l} 
& & \psi S_{5}^{1} \mathrm{~K} 3 S_{10}^{1} \\
q_{2}=N_{P} & M 2 & \sim \sim \alpha_{2} \sim \\
q_{3}=-N_{F} & M 2 & \sim \sim \alpha_{3} \sim \\
2 J_{L}^{3}=n_{p} & p & \bigcirc \sim \sim \sim \\
p^{1}=-n_{f} & m 5 & \bigcirc \sim \bigcirc \sim
\end{array}
$$

where $J_{L}^{3}$ is the $\mathrm{SU}(2)_{L} \subset \mathrm{SO}(4)$ charge. This can be thought of as a $5 \mathrm{D}$ small black ring (internal directions are $S_{5}^{1}, \mathrm{~K} 3, S_{10}^{1}$ ), and is simply a special case of the configurations considered in [10-13]. So, by the (string corrected) 4D-5D connection, there is a way to continuously deform this $5 \mathrm{D}$ ring, by way of a Taub-NUT geometry, into a 5D black string which from the $4 \mathrm{D}$ point of view is a black hole. The entropy of the $5 \mathrm{D}$ ring and that of the 4D hole are identical since entropy cannot change in such an adiabatic process. In this process, the role of the Taub-NUT is just a "catalyst" to wind the ring around the M-circle and it can be removed when we have reached the $4 \mathrm{D}$ configuration, since the entropy is independent of the D6 charge. Practically, one can say that we can reinterpret the contractible circle $\psi$ as a non-contractible M-circle, if the relation between $4 \mathrm{~d}$ and $5 \mathrm{~d}$ charges (eq. (3.5) below) is correctly taken into account. Compactifying now on this non-contractible M-circle, we obtain a 4D small non-rotating black hole:

$$
\begin{array}{ll|l|l} 
& & S_{5}^{1} \mathrm{~K} 3 S_{10}^{1} \\
\cline { 2 - 3 }=N_{P} & D 2 & \sim \alpha_{2} \sim \\
q_{3}=-N_{F} & D 2 & \sim \alpha_{3} \sim \\
q_{0}=n_{p} & d 0 & \sim \sim \sim \\
p^{1}=-n_{f} & d 4 & \sim \bigcirc \sim
\end{array}
$$


where we used the relation between $5 \mathrm{D}$ and $4 \mathrm{D}$ charges [14, 13, 18, 10],

$$
p_{4 D}^{A}=p_{5 D}^{A}, \quad q_{A}^{4 D}=q_{A}^{5 D}-3 D_{A B C} p^{B} p^{C}=q_{A}^{5 D}, \quad q_{0}^{4 D}=2 J_{L}^{3} .
$$

The reserved index 1 is now for the 2 -cycle $T^{2}=S_{5}^{1} \times S_{10}^{1}$.

The object with charges (3.4) does not have a classical horizon, but develops a finite

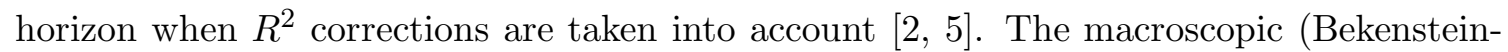
Hawking-Wald) entropy is

$$
S_{\text {macro }}=2 \pi \sqrt{\frac{\hat{q}_{0} c_{2 A} p^{A}}{6}}, \quad \hat{q}_{0}=q_{0}+\frac{1}{12} \hat{D}^{A B} q_{A} q_{B}, \quad D_{A B}=D_{A B C} p^{C} .
$$

Here $c_{2 A}$ are the coefficients of the second Chern class, and $D_{A B C}=\frac{1}{6} C_{A B C}$, where $C_{A B C}$ is the triple intersection number. The matrix $\hat{D}^{A B}$ is the inverse of $D_{A B}$ in the subspace orthogonal to its kernel.

In the present case, $c_{2, A=1}=24$ and $C_{1 a b}=C_{a b}, a, b=1, \ldots, 23$ with the only relevant values given in (3.2). Therefore, the macroscopic entropy (3.6) is computed as

$$
S=4 \pi \sqrt{q_{0} p^{1}+q_{2} q_{3}}=4 \pi \sqrt{-N_{P} N_{F}-n_{p} n_{f}}
$$

Thus we reproduced the microscopic entropy (eq. 2.10) of the D1-D5-J system from the macroscopic entropy of the 4D small black hole. This supports the validity of the 4D$5 \mathrm{D}$ connection that we used to arrive at the $4 \mathrm{D}$ configuration (3.4), even when stringy corrections are crucial.

\section{Conclusion and discussion}

The D1-D5-J system is expected to develop a finite horizon once stringy $R^{2}$ corrections to the supergravity action are taken into account, and become a 5D small black ring. We clarified the limit, $|J| \gg \sqrt{N}$, in which the D1-D5-J system can be regarded as a ring with a well-defined profile, and showed that we can relate this system by duality transformations and the 4D-5D connection to a 4D small non-rotating black hole, whose horizon structure is well-understood. This gives an indirect evidence that event horizon appears in the 5D small black ring by stringy corrections. We also checked that the entropy of the $4 \mathrm{D}$ small black hole agrees with the microscopic entropy of the D1-D5-J system, which can be regarded as a consistency check.

A novel idea for understanding black hole physics was put forward by Mathur and collaborators (for a review see [30]), who conjectured that a black hole is not a singularity surrounded by empty space and horizon, but an ensemble of smooth but complicated (possibly quantum) geometries inside the stretched horizon. This bold conjecture has been established for the BPS D1-D5 system, for which microstate geometries have been explicitly written down at the supergravity level [31, 32]..$^{5}$ In this Mathur picture, the entropy of a black hole can be estimated from the area of the "stretched horizon," defined as the surface

\footnotetext{
${ }^{5}$ The supergravity microstate solutions obtained in 31 , 32 include fluctuations in the noncompact $\mathbb{R}^{4}$ directions, but not in the internal $T^{4}$ or K3 directions.
} 
on which the microstate geometries start to differ from each other. In [8], an ensemble of D1-D5 microstate geometries with angular momentum $J$ were studied, and the entropy was correctly estimated to be $\sim \sqrt{N_{1} N_{5}-|J|}$ from this "stretched horizon" area. It is interesting to study the relation between their "stretched horizon" and the non-vanishing horizon that we argued to appear in the 5D small black ring by stringy corrections.

The above definition of the stretched horizon by Mathur is a qualitative one, and in order to determine the entropy of a black hole from an area law including the numerical factor, it is desirable to define the stretched horizon in a more precise way. It is also interesting to study the relation between this definition of the stretched horizon and other definitions, for example, as the distance at which a probe D-brane starts to become indistinguishable from the D-branes that compose the background black hole, due to thermal effect [33].

Another aspect of the D1-D5-J system is that, being dual to a configuration of fundamental heterotic string, it is an example of systems for which the microscopic entropy can be computed very precisely [2, 34]. It is interesting to analyze this system further to study the recently proposed relation between the black hole partition function and the topological string partition function [35, 36, 34].

\section{Acknowledgments}

It is pleasure to thank Atish Dabholkar and Ashoke Sen for helpful conversations. We also would like to thank Per Kraus for useful comments on the early draft of this note. N.I. would like to thank friends in Harvard and Caltech string theory group for their very nice hospitality where this work was done. The work of M.S. was supported in part by Department of Energy grant DE-FG03-92ER40701 and a Sherman Fairchild Foundation postdoctoral fellowship. Finally we are happy to thank the people of India for their generosity.

\section{References}

[1] A. Strominger and C. Vafa, Microscopic origin of the Bekenstein-Hawking entropy, Phys. Lett. B 379 (1996) 99 hep-th/9601029.

[2] A. Dabholkar, Exact counting of black hole microstates, Phys. Rev. Lett. 94 (2005) 241301 hep-th/0409148

[3] G. Lopes Cardoso, B. de Wit and T. Mohaupt, Corrections to macroscopic supersymmetric black-hole entropy, Phys. Lett. B 451 (1999) 309 hep-th/9812082]; Deviations from the area law for supersymmetric black holes, Fortschr. Phys. 48 (2000) 49 hep-th/9904005;

Macroscopic entropy formulae and non-holomorphic corrections for supersymmetric black holes, Nucl. Phys. B 567 (2000) 87 hep-th/9906094; Area law corrections from state counting and supergravity, Class. and Quant. Grav. 17 (2000) 1007 hep-th/9910179;; T. Mohaupt, Black hole entropy, special geometry and strings, Fortschr. Phys. 49 (2001) 3 hep-th/0007195.

[4] A. Sen, Extremal black holes and elementary string states, Mod. Phys. Lett. A 10 (1995) 2081 hep-th/9504147. 
[5] A. Dabholkar, R. Kallosh and A. Maloney, A stringy cloak for a classical singularity, JHEP 12 (2004) 059 hep-th/0410076.

[6] A. Sen, How does a fundamental string stretch its horizon?, JHEP 05 (2005) 059 hep-th/0411255;

V. Hubeny, A. Maloney and M. Rangamani, String-corrected black holes, JHEP 05 (2005) 035 hep-th/0411272;

D. Bak, S. Kim and S.-J. Rey, Exactly soluble BPS black holes in higher curvature $N=2$ supergravity, hep-th/0501014;

A. Sen, Black holes, elementary strings and holomorphic anomaly, JHEP 07 (2005) 063 hep-th/0502126; Black holes and the spectrum of half-BPS states in $N=4$ supersymmetric string theory, hep-th/0504005; Stretching the horizon of a higher dimensional small black hole, JHEP 07 (2005) 073 hep-th/0505122.

[7] O. Lunin and S.D. Mathur, Metric of the multiply wound rotating string, Nucl. Phys. B 610 (2001) 49 hep-th/0105136.

[8] O. Lunin and S.D. Mathur, Statistical interpretation of Bekenstein entropy for systems with a stretched horizon, Phys. Rev. Lett. 88 (2002) 211303 hep-th/0202072.

[9] H. Elvang, R. Emparan, D. Mateos and H.S. Reall, A supersymmetric black ring, Phys. Rev. Lett. 93 (2004) 211302 hep-th/0407065.

[10] D. Gaiotto, A. Strominger and X. Yin, New connections between $4 D$ and $5 D$ black holes, hep-th/0503217.

[11] H. Elvang, R. Emparan, D. Mateos and H.S. Reall, Supersymmetric $4 D$ rotating black holes from $5 D$ black rings, hep-th/0504125.

[12] D. Gaiotto, A. Strominger and X. Yin, 5D black rings and $4 D$ black holes, hep-th/0504126.

[13] I. Bena, P. Kraus and N.P. Warner, Black rings in Taub-NUT, hep-th/0504142.

[14] I. Bena and P. Kraus, Microscopic description of black rings in AdS/CFT, JHEP 12 (2004) 070 hep-th/0408186.

[15] M. Cyrier, M. Guica, D. Mateos and A. Strominger, Microscopic entropy of the black ring, Phys. Rev. Lett. 94 (2005) 191601 hep-th/0411187.

[16] J.M. Maldacena, A. Strominger and E. Witten, Black hole entropy in M-theory, JHEP 12 (1997) 002 hep-th/9711053.

[17] M. Guica, L. Huang, W. Li and A. Strominger, $R^{2}$ corrections for $5 D$ black holes and rings, hep-th/0505188.

[18] I. Bena, $R^{2}$ corrections to black ring entropy, hep-th/0506015.

[19] P. Kraus and F. Larsen, Microscopic black hole entropy in theories with higher derivatives, hep-th/0506176.

[20] D. Mateos, S. Ng and P.K. Townsend, Supercurves, Phys. Lett. B 538 (2002) 366 hep-th/0204062.

[21] E. Witten, String theory dynamics in various dimensions, Nucl. Phys. B 443 (1995) 85 hep-th/9503124.

[22] E. Kiritsis, Heterotic/type-II duality and its field theory avatars, AIP Conf. Proc. 490 (1999) 217. 
[23] C. Vafa, Gas of D-branes and hagedorn density of BPS states, Nucl. Phys. B 463 (1996) 415 hep-th/9511088.

[24] J.G. Russo and L. Susskind, Asymptotic level density in heterotic string theory and rotating black holes, Nucl. Phys. B 437 (1995) 611 hep-th/9405117.

[25] G.T. Horowitz and A. Sen, Rotating black holes which saturate a bogomol'nyi bound, Phys. Rev. D 53 (1996) 808 hep-th/9509108.

[26] O. Lunin and S.D. Mathur, Three-point functions for $M(N) / S(N)$ orbifolds with $N=4$ supersymmetry, Commun. Math. Phys. 227 (2002) 385 hep-th/0103169.

[27] C.M. Hull and P.K. Townsend, Unity of superstring dualities, Nucl. Phys. B 438 (1995) 109 hep-th/9410167.

[28] J. Polchinski, String theory, 2: Superstring theory and beyond, Cambridge University Press, Cambridge 1998.

[29] P.S. Aspinwall and D.R. Morrison, String theory on K3 surfaces, hep-th/9404151.

[30] S.D. Mathur, The fuzzball proposal for black holes: an elementary review, Fortschr. Phys. 53 (2005) 793 hep-th/0502050.

[31] O. Lunin and S.D. Mathur, AdS/CFT duality and the black hole information paradox, Nucl. Phys. B 623 (2002) 342 hep-th/0109154.

[32] O. Lunin, J. Maldacena and L. Maoz, Gravity solutions for the D1-D5 system with angular momentum, hep-th/0212210.

[33] N. Iizuka, D. Kabat, G. Lifschytz and D.A. Lowe, Probing black holes in non-perturbative gauge theory, Phys. Rev. D 65 (2002) 024012 hep-th/0108006.

[34] A. Dabholkar, F. Denef, G.W. Moore and B. Pioline, Exact and asymptotic degeneracies of small black holes, hep-th/0502157.

[35] H. Ooguri, A. Strominger and C. Vafa, Black hole attractors and the topological string, Phys. Rev. D 70 (2004) 106007 hep-th/0405146.

[36] E.P. Verlinde, Attractors and the holomorphic anomaly, hep-th/0412139. 